\title{
Blocking TNF- $\alpha$ in mice reduces colorectal carcinogenesis associated with chronic colitis
}

\author{
Boryana K. Popivanova, ${ }^{1}$ Kazuya Kitamura, ${ }^{2}$ Yu Wu, ${ }^{1}$ Toshikazu Kondo, ${ }^{3}$ Takashi Kagaya, ${ }^{2}$ \\ Shiuchi Kaneko,2 Masanobu Oshima, ${ }^{4}$ Chifumi Fujii, ${ }^{1}$ and Naofumi Mukaida1
}

1Division of Molecular Bioregulation, Cancer Research Institute, and 2Department of Disease Control and Homeostasis, Graduate School of Medical Science, Kanazawa University, Kanazawa, Japan. ${ }^{3}$ Department of Legal Medicine, Wakayama Medical University, Wakayama, Japan. ${ }^{4}$ Division of Genetics, Cancer Research Institute, Kanazawa University, Kanazawa, Japan.

\begin{abstract}
The inflammatory bowel disease ulcerative colitis (UC) frequently progresses to colon cancer. To understand the mechanisms by which UC patients develop colon carcinomas, we used a mouse model of the disease whereby administration of azoxymethane (AOM) followed by repeated dextran sulfate sodium (DSS) ingestion causes severe colonic inflammation and the subsequent development of multiple tumors. We found that treating WT mice with AOM and DSS increased TNF- $\alpha$ expression and the number of infiltrating leukocytes expressing its major receptor, p55 (TNF-Rp55), in the lamina propria and submucosal regions of the colon. This was followed by the development of multiple colonic tumors. Mice lacking TNF-Rp55 and treated with AOM and DSS showed reduced mucosal damage, reduced infiltration of macrophages and neutrophils, and attenuated subsequent tumor formation. WT mice transplanted with TNF-Rp55-deficient bone marrow also developed significantly fewer tumors after AOM and DSS treatment than either WT mice or TNF-Rp55-deficient mice transplanted with WT bone marrow. Furthermore, administration of etanercept, a specific antagonist of TNF- $\alpha$, to WT mice after treatment with AOM and DSS markedly reduced the number and size of tumors and reduced colonic infiltration by neutrophils and macrophages. These observations identify TNF- $\alpha$ as a crucial mediator of the initiation and progression of colitis-associated colon carcinogenesis and suggest that targeting TNF- $\alpha$ may be useful in treating colon cancer in individuals with UC.
\end{abstract}

\section{Introduction}

Ulcerative colitis (UC) is an inflammatory bowel disease characterized by pathological mucosal damage and ulceration, which can involve the rectum and extend proximally (1). The incidence of UC in the United States is about 4-12 per 100,000 and has risen in recent decades. UC typically presents as a relapsing disorder marked by attacks of bloody mucoid diarrhea that sometimes persists for months, only to recur after an asymptomatic interval of months to years (1). UC consistently manifests DNA damage with microsatellite instability in mucosal cells (2). Thus, repetitive relapses and remissions can frequently cause epithelial dysplasia and can eventually progress to invasive cancer (3). Indeed, involvement of the entire colon for longer than 10 years predisposes UC patients to colon cancer, and the risk of cancer is 20- to 30 -fold higher in these patients than in a control population (4). Thus, it is desirable to develop measures to prevent cancer development in UC patients based on an understanding of the pathogenesis of colon carcinogenesis in UC at molecular and cellular levels.

Oral administration of dextran sulfate sodium (DSS) solution to rodents is widely employed to recapitulate human UC, because it can cause acute inflammatory reaction and ulceration in the entire colon similar to that observed in UC patients (5). Moreover, repeated oral DSS ingestion alone can cause colon carcinoma in a proportion of mice when the ingestion is of 7 days' duration

Nonstandard abbreviations used: AOM, azoxymethane; DSS, dextran sulfate sodium; GSK, glycogen synthase kinase; IKK $\beta$, IKB kinase $\beta$; KC, keratinocyte chemoattractant; MCP-1, monocyte chemoattractant protein-1; TNF-Rp55, TNF receptor $\mathrm{p} 55$; UC, ulcerative colitis.

Conflict of interest: The authors have declared that no conflict of interest exists. Citation for this article: J. Clin. Invest. 118:560-570 (2008). doi:10.1172/JCI32453. and is repeated 9 times (6). These observations suggest that the inflammatory response alone can cause colon carcinoma. Azoxymethane $(\mathrm{AOM})$ is also frequently used to induce tumors in the distal colon of rodents by causing $\mathrm{O}^{6}$-methyl-guanine formation (7). A prior administration of AOM can accelerate and increase the incidence of DSS-induced colon carcinogenesis, as evidenced by the very high incidence of colon cancer (nearly 100\%) after 3 subsequent rounds of DSS ingestion (8). Inactivation of the IкB/NF-кB pathway has been associated with reducing colon carcinogenesis induced by the combined treatment of AOM and DSS (9). This observation may mirror the enhanced NF-KB activation seen in human colon adenoma and cancer tissues $(10,11)$. This suggests that induction of NF- $\mathrm{KB}$ by inflammatory stimuli may participate in colon carcinogenesis. However, it still remains to be determined, which molecule(s), if any, aberrantly enhances NF-KB activation during the course of colon carcinogenesis.

NF- $\mathrm{KB}$ activation is required for the expression of many proinflammatory molecules including cytokines and adhesion molecules (12). Among these cytokines, TNF- $\alpha$ can further augment NF- $\mathrm{B}$ activation in various cell types after binding to either TNF receptor p55 (TNF-Rp55) or TNF-Rp75 (13). Since TNF-Rp55 is widely expressed on almost all cell types except erythrocytes (14), TNF-Rp55 deficiency has profound effects on endotoxin shock (15) and the skin wound healing process (16). TNF- $\alpha$ was originally identified as a mediator responsible for endotoxin-induced tumor necrosis (17) and was utilized for the treatment of patients with advanced localized solid tumors (18). On the contrary, we observed that liver and lung metastasis were depressed in TNFRp55-deficient (TNF-Rp55-/-) mice $(19,20)$, suggesting a crucial contribution of the TNF- $\alpha /$ TNF-Rp55 axis to the development of metastasis. Moreover, TNF- $\alpha$-deficient mice developed fewer 
A

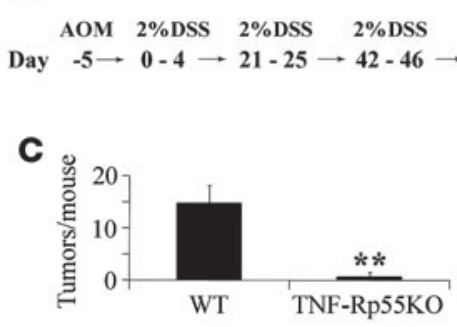

B

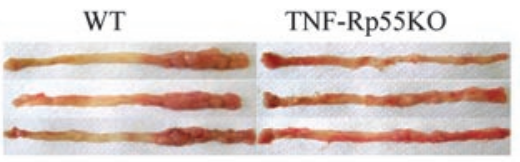

D

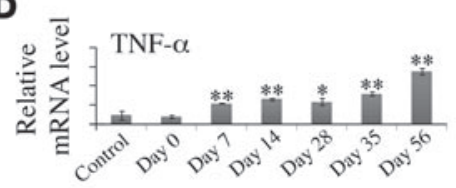

E

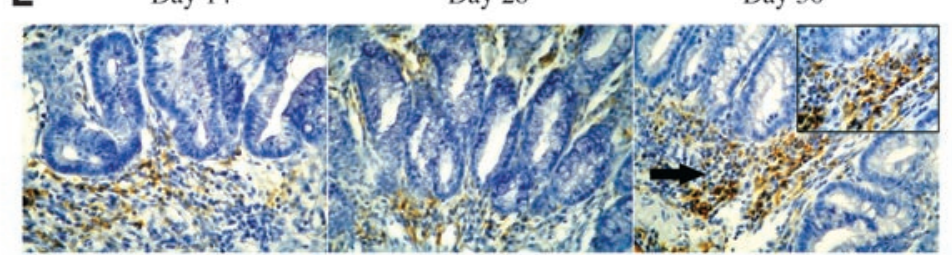

$\mathbf{F}$

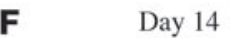

Day 14

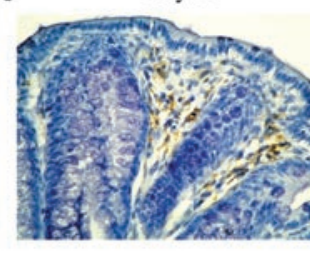

Day 28

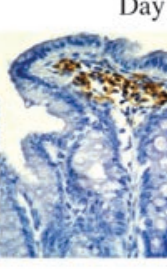

Day 56
Day 56

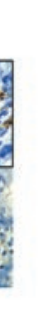
.

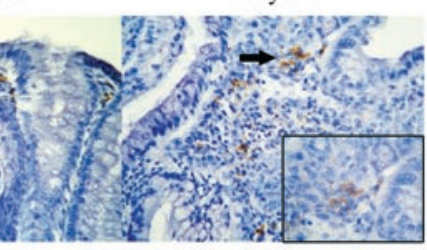

\section{Figure 1}

Tumor formation in WT and TNF-Rp55 ${ }^{--}$(TNFRp55KO) mice after AOM and DSS treatment. (A) Schematic overview of this colon carcinogenesis model. (B) Macroscopical changes in colon. Colons were removed at day 56 from treated WT and TNF-Rp55 $5^{--}$mice, and representative results from 10 independent animals are shown here. (C) The numbers of tumors. Colons were removed at day 56 to determine the numbers of macroscopic tumors. Each value represents the mean \pm SD ( $n=10$ animals). ${ }^{* *} P<0.01$ versus WT. (D). TNF- $\alpha$ gene expression in the colons of WT mice. The levels of TNF- $\alpha$ mRNA were quantified by quantitative RT-PCR as described in Methods, and normalized to the level of GAPDH mRNA. ${ }^{*} P<0.05$, ${ }^{* \star} P<0.01$ versus untreated (control) mice. (E and F) Immunohistochemical detection of TNF- $\alpha$ and TNF-Rp55 in colons. Colons were obtained from WT mice at the indicated time intervals; insets are higher magnification of the positively stained cells as indicated by arrows. Representative results from 6 independent experiments are shown here (original magnification, $\times 400 ; \times 1,000$ [insets]). tumors than WT mice when exposed to several types of carcinogens (21). Thus, the TNF- $\alpha$ /TNF-Rp55 axis may actually promote carcinogenesis and its progression.

Here we investigated the roles of the TNF- $\alpha / T N F-R p 55$ axis in colon carcinogenesis induced by the combined treatment with AOM and DSS by utilizing TNF-Rp55/- mice and a human TNFspecific antagonist, etanercept, which can block mouse TNF- $\alpha$ activities (22). Our experiments revealed that the combined treatment with AOM and DSS induced the intracolonic expression of TNF- $\alpha$, which in turn regulated the trafficking of inflammatory cells, a major source of COX-2, thereby resulting in the development and progression of colon cancer.

\section{Results}

Enhanced TNF expression in the colon during the course of colon carcinogenesis. Consistent with previous reports $(8,9)$, a single intraperitoneal injection of the carcinogen AOM, followed by 3 rounds of $2 \%$ DSS intake induced the development of multiple tumors in the middle to distal colon of WT mice (Figure 1, B and C). The essential involvement of a transcription factor, NF- $\kappa \mathrm{B}$, in this colon carcinogenesis model (9) prompted us to investigate the intracolonic expression of TNF- $\alpha$ and its receptor because TNF- $\alpha$ is a potent activator of NF- $\kappa \mathrm{B}(13,14)$. TNF- $\alpha$ mRNA was faintly expressed in untreated WT mice, and AOM treatment alone did not enhance TNF- $\alpha$ mRNA expression, but subsequent DSS intake augmented TNF- $\alpha$ mRNA expression (Figure 1D). We also detected TNF- $\alpha$ protein expression by immunohistochemical analysis mainly in mononuclear cells present in lamina propria and submucosal regions (Figure 1E). Similarly, immunoreactive TNF- $\alpha$ protein was detected in the colons of patients with active UC and advanced colorectal cancer, but not in normal mucosa (Supplemental Figure 1; supplemental material available online with this article; doi:10.1172/JCI32453DS1). Immunohistochemical analysis detected the major receptor for TNF- $\alpha$, TNF-Rp55, predominantly in leukocytes infiltrating the lamina propria and submucosal regions of the colon during the course of this colon carcinogenesis model (Figure $1 \mathrm{~F}$ ).

Reduced tumor incidence in TNF-Rp55-/- mice. In order to clarify the role of TNF-Rp55 in this colon carcinogenesis model, we treated both WT and TNF-Rp55/- mice with AOM and DSS in the same manner. During the course of AOM and DSS treatment, WT mice exhibited profound body weight loss and bloody diarrhea, whereas TNF-Rp55/- mice had less body weight loss and did not have bloody diarrhea (data not shown). There were no apparent differences in macroscopical and microscopical appearance of the colon of untreated WT and TNF-Rp55/- mice (Figure 2A). In treated WT mice, edema and hyperemia of the middle to distal colon became evident after day 7, and multiple tumors developed in the same region after day 28 , whereas these morphological changes were rare in AOM and DSS-treated TNF-Rp55/- mice (data not shown). Histological analysis consistently demonstrated massive infiltration of leukocytes into the mucosa and edema of the submucosa, with loss of entire crypts and surface epithelium by day 7, particularly in the middle to distal colon of WT mice (Figure 2A). At day 14, mucosal inflammatory cell infiltration persisted and was accompanied by dysplastic glands with hyperchromatic nuclei, decreased mucin production, and dystrophic goblet cells. By days 28 to 35, macroscopically visible adenocarcinomatous lesions developed, and their size and numbers increased progressively thereafter. Moreover, $\beta$-catenin accumulated in the nuclei of the tumor cells after day 28 (Figure 2B). On the contrary, TNF-Rp55/- mice displayed much milder inflammation of the colon during the course of DSS intake and developed fewer adenocarcinomatous lesions and less nuclear $\beta$-catenin accumulation (Figure 1C and Figure 2, 


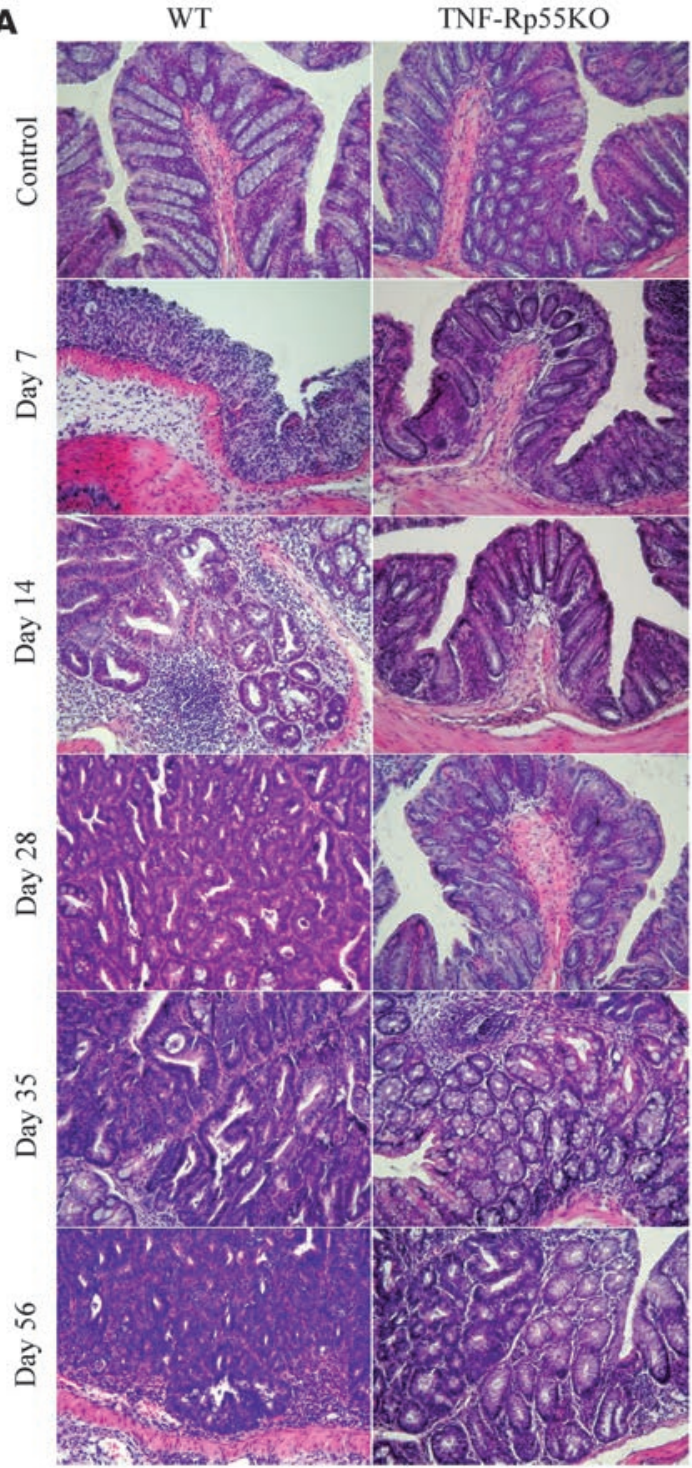

A

WT

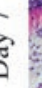

\section{E}

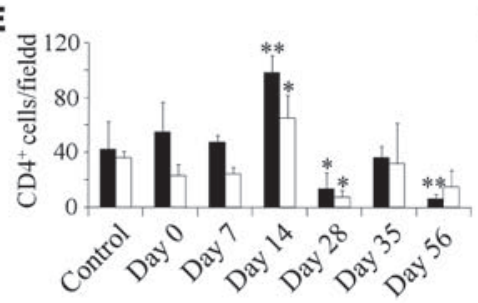

$\mathbf{F}$



Day 28



WT

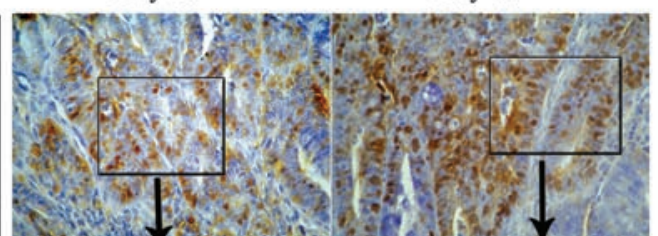

$\mathrm{KO}$
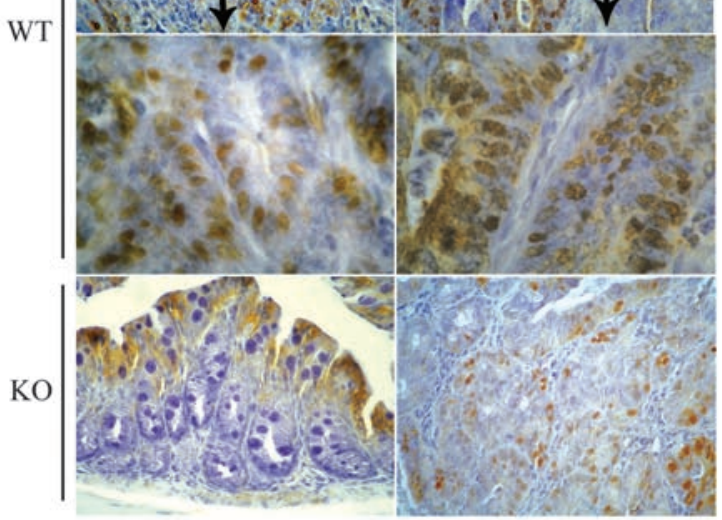

C

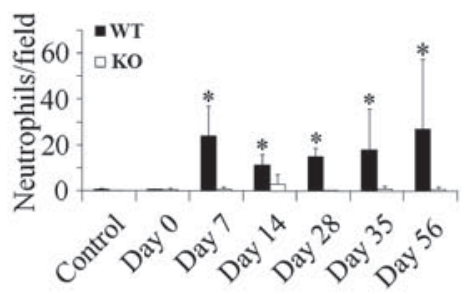

D
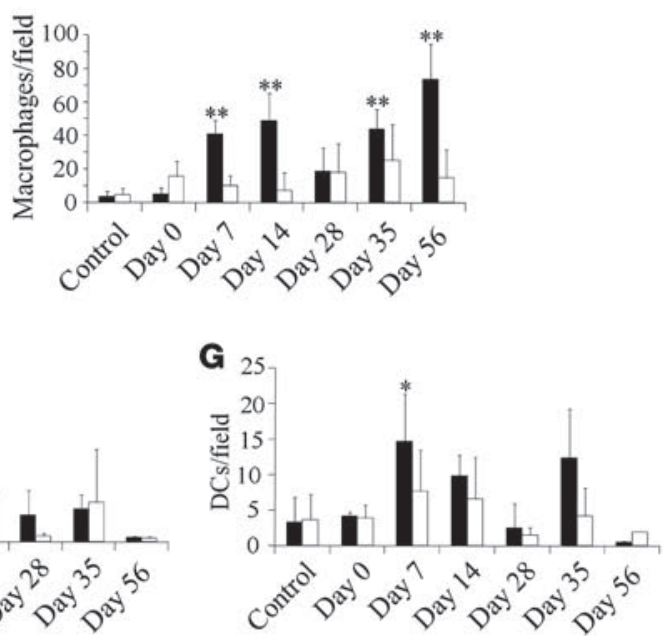

Figure 2

Microscopical analysis of colon tissues. (A) Colons were removed at the indicated time intervals, fixed, and stained with hematoxylin and eosin. Representative results from 5 mice are shown here. Original magnification, $\times 200$. (B) Immunohistochemical staining for $\beta$-catenin. Colons were removed at the indicated time intervals from WT and TNF-Rp55 ${ }^{-1-}$ (TNF-Rp55KO) mice and immunostained with anti- $\beta$-catenin antibody as described in Methods. Boxed areas in the left panels are shown at higher magnification in the middle panels. Representative results from 3 independent animals are shown here. Original magnification, $\times 400$ (top and bottom rows), $\times 1,000$ (middle row). (C-G) The numbers of myeloperoxidase- (C), F4/80- (D), CD4- (E), CD8- (F), and DEC205-positive cells (G) were counted as described in Methods and are shown here. All values represent the mean $\pm \mathrm{SD}\left(n=10\right.$ animals). ${ }^{*} P<0.05,{ }^{* *} P<0.01$ versus untreated (control) WT mice.

A and B). Moreover, the numbers of apoptotic cells were transiently increased at day 7 in WT but not TNF-Rp55/-- mice, as revealed by TUNEL assay (Supplemental Figure 2). However, the incidence of apoptotic cells detected in TNF-Rp55/- mice was similar in level to those in WT mice except on day 7 (Supplemental Figure 2). These observations suggest a crucial role in this model for TNF-Rp55mediated signals in the development of chronic inflammation and colon carcinoma but not in the apoptotic reactions. 

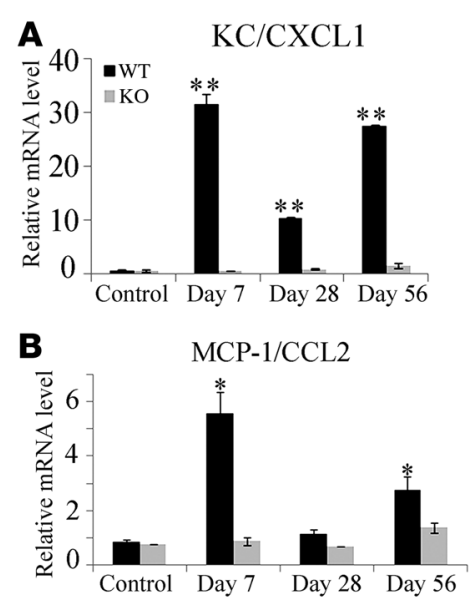

Inflammatory cell infiltration. We next proceeded to identify the types of cells that were decreased in the absence of TNF-Rp55 by immunohistochemical analysis. In treated WT mice, neutrophils and macrophages infiltrated into lamina propria and submucosa after day 7 and persisted until day 56 (Supplemental Figure 3). In addition, after day 7 aggregates of CD4-positive lymphocytes and DEC205-positive dendritic cells infiltrated into the lamina propria and submucosa (Supplemental Figure 3). In AOM and DSS-treated TNF-Rp55/- mice, infiltration by both neutrophils and macrophages was markedly decreased, whereas lymphocyte and dendritic cell infiltration was minimally affected (Figure 2, $\mathrm{C}-\mathrm{G})$. TNF- $\alpha$ can augment the expression of the chemokines keratinocyte chemoattractant/CXCL1 (KC/CXCL1) and monocyte chemoattractant protein-1/CCL2 (MCP-1/CCL2), which are chemotactic for neutrophils and macrophages, respectively $(23,24)$. Indeed, after day 7 , gene expression of both chemokines was enhanced in WT mice, but their expression was consistently depressed in TNF-Rp55/- mice (Figure 3). These observations suggest that TNF-Rp55-mediated signals were responsible for the trafficking of neutrophils and macrophages, at least in part by enhancing the expression of chemokines. Moreover, it is plausible that bone marrow-derived cells, neutrophils and macrophages, can be crucially involved in this colon carcinogenesis model. In order to address the contribution of bone marrow-derived cells, we treated various bone marrow chimeric mice with the same combination of AOM and DSS. TNF-Rp55/- mice transplanted with WT-derived bone marrow cells developed tumors at a similar level as WT mice transplanted with WT-derived bone marrow cells, but at a higher level than either WT or TNF-Rp55/- mice transplanted

\section{Figure 3}

Chemokine gene expression in the colons. Quantitative RT-PCR was performed on total RNAs extracted from the colons at the indicated time intervals as described in Methods. The levels of KC/CXCL1 (A) and MCP-1/CCL2 (B) mRNA were normalized to GAPDH mRNA levels. Representative results from 5 independent experiments are shown in here. ${ }^{*} P<0.05,{ }^{*} P<0.01$ versus untreated (control) mice. with TNF-Rp55/- mouse-derived bone marrow cells (Figure 4). These observations suggest that TNF-Rp55-mediated signals act mainly on bone marrow, but not non-bone marrow-derived cells in this carcinogenesis model.

Reduced COX-2 expression in TNF-Rp55 ${ }^{-/-}$mice. Accumulating evidence indicates the causal involvement of COX-2 in colon carcinogenesis. Hence, we examined COX-2 mRNA expression by real-time RT-PCR. After day 7, intracolonic COX-2 expression was markedly enhanced in treated WT but not TNF-Rp55/- mice (Figure 5A). COX-2 protein was detected mainly in infiltrating inflammatory cells (Figure 5B), and the numbers of COX-2-positive cells increased from day 7 to day 56 in WT but not TNF-Rp55/- mice (Figure 5, B and C). Double-color immunofluorescence analysis detected COX-2 protein in F4/80-positive macrophages and to a lesser extent in Ly-6G-positive neutrophils (Figure 5D). These observations suggest that in the absence of TNF-Rp55 the infiltration of macrophages and neutrophils, which are a major source of COX-2, was reduced, leading to decreased COX-2 expression.

Effect of TNF- $\alpha$ antagonist, etanercept, on tumor formation in WT mice. Because the human TNF antagonist, etanercept, can inhibit the biological activity of murine TNF (22), we explored its effects on tumor progression by administering it to mice from day 56 to day 60, after the AOM and 3 cycles of DSS treatments (Figure 6A). Compared with the vehicle-treated group, etanercept treatment reduced the numbers and size of macroscopical tumors remarkably when administered even over this short and delayed time period (Figure 6 , B-D). Concomitantly, etanercept treatment reduced intracolonic infiltration by inflammatory cells, particularly neutrophils and macrophages (Figure 6, E and F, and Supplemental Figure 4),

\section{Figure 4}

Colon tumor formation in bone marrow chimeric mice. Bone marrow chimeric mice were generated and subjected to AOM+DSS treatment as described in Methods. Colons were removed at day 56, and the tumor numbers were determined macroscopically. The bars represent the median of each group; each symbol represents the tumor numbers of each animal. ${ }^{* *} P<0.01$.






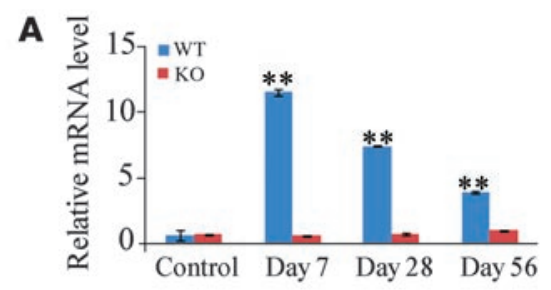

B

Day 0

Day 7

Day 28

Day 56
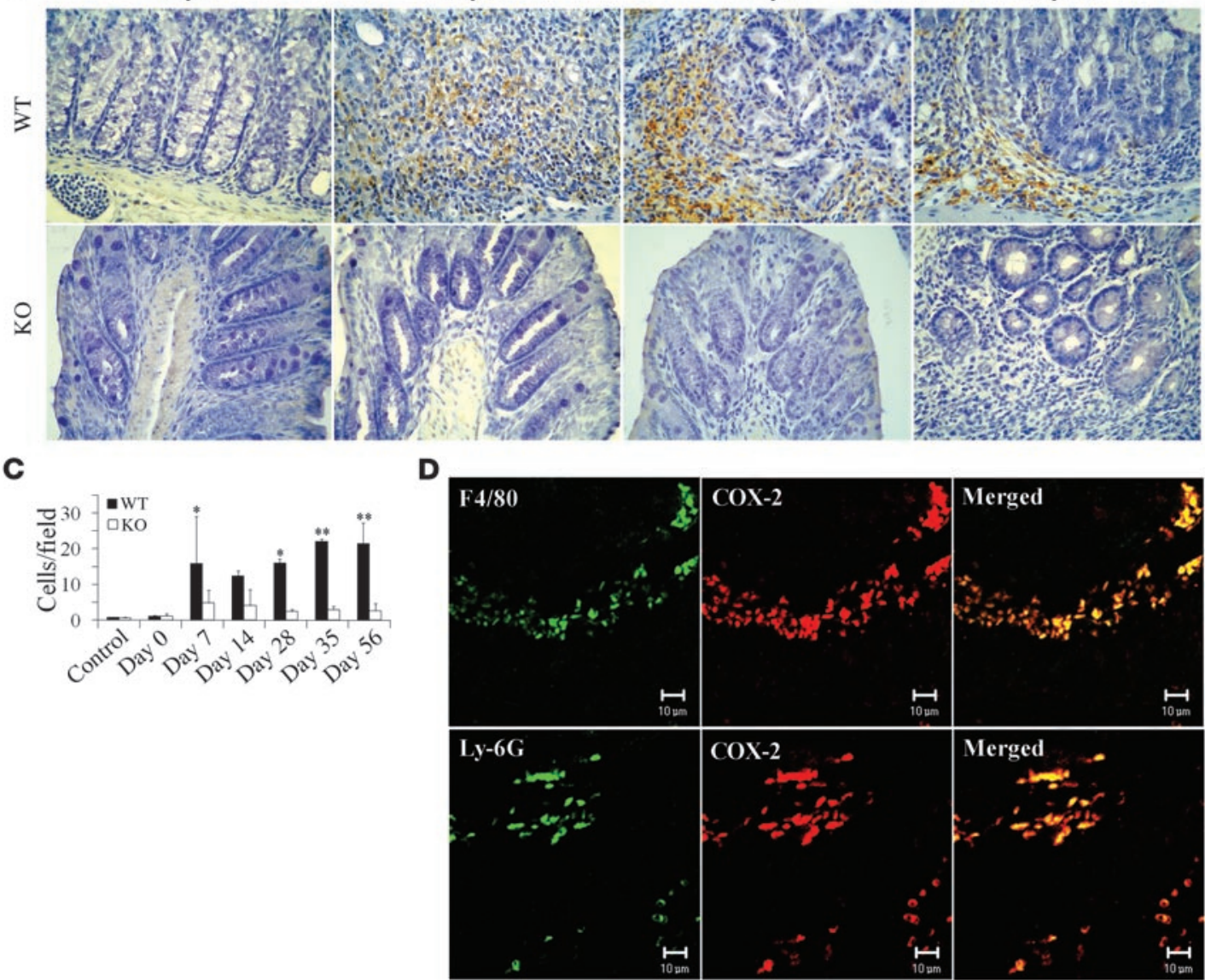

Figure 5

COX-2 expression in the colons. (A) Quantitative RT-PCR was performed on total RNAs extracted from the colons at the indicated time intervals as described in Methods. The levels of COX-2 mRNA were normalized to the levels of GAPDH mRNA. ${ }^{* *} P<0.01$ versus untreated (control) mice. (B-D) Immunohistochemical and immunofluorescence detection of COX-2-expressing cells. Colons were obtained from WT mice at the indicated time intervals and processed for immunohistochemical analysis using anti-COX-2 antibodies, and representative results from 5 independent animals are shown in $\mathbf{B}$. The numbers of COX-2-expressing cells were determined as described in Methods and are shown in $\mathbf{C}$ and expressed as mean $\pm \mathrm{SD}$. ${ }^{*} P<0.05$, ${ }^{* *} P<0.01$ versus untreated. Double-color immunofluorescence analysis was performed with the combination of anti-COX-2 and anti-F4/80 (D, top row) or that of anti-COX-2 and anti-Ly6G antibodies (D, bottom row). Representative results from 5 independent experiments are shown here. Original magnification, $\times 400$ (B); $\times 800$ (D). Scale bars, $10 \mu \mathrm{m}$.

together with a decrease in mRNA levels of the neutrophil-tropic chemokine, KC/CXCL1, and the macrophage-tropic chemokine, MCP-1/CCL2 (Figure 6, G and H). Furthermore, etanercept reduced COX-2 mRNA expression (Figure 7A) and the numbers of COX-2 expressing cells (Figure 7, B and C). Because COX-2-derived $\mathrm{PGE}_{2}$ is an important stimulant of tumor angiogenesis (25), we next examined the effect of etanercept on the intratumoral vascular density by immunostaining with anti-CD31 antibody. At 56 and 67 days after the initiation of DSS intake, WT mice exhibited a marked increase in vascular densities, and this increment was markedly depressed by etanercept (Figure 7, D and E).
$\mathrm{PGE}_{2}$ has also been reported to have direct effects on the $\beta$-catenin axis (26). This prompted us to evaluate the state of $\beta$-catenin in the tumors of mice treated with etanercept. Indeed, etanercept also decreased the nuclear accumulation of $\beta$-catenin at the tumor sites (Figure 8, A and B). The amounts of unphosphorylated (active) $\beta$-catenin protein were increased in WT mice at days 56 and 67, and etanercept markedly reduced this increase (Figure 8C). Moreover, etanercept markedly decreased the numbers of cytokeratin 20-positive cells (Figure 8, A and D), which represent colon adenocarcinoma cells (27). Takahashi and colleagues observed that in these AOM-induced tumors, the $\beta$-catenin 

A AOM 2\%DSS 2\%DSS 2\%DSS Etanercept Sacrifice Day $-5 \longrightarrow 0-4 \longrightarrow 21-25 \longrightarrow 42-46 \longrightarrow 56-60 \longrightarrow 67$

B
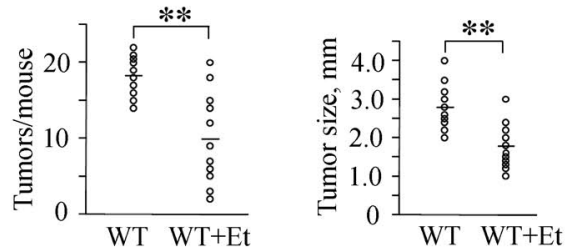
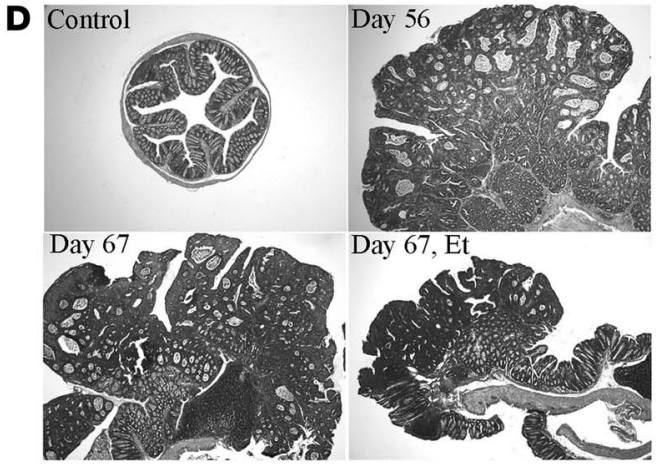

Day 67, Et

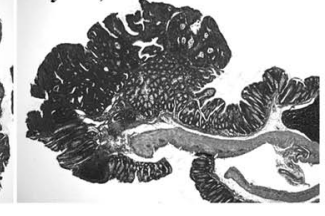

E

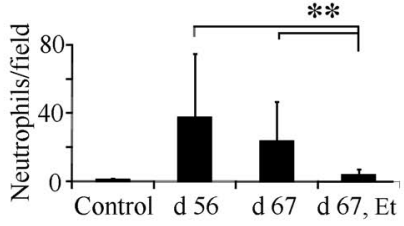

$\mathbf{F}$

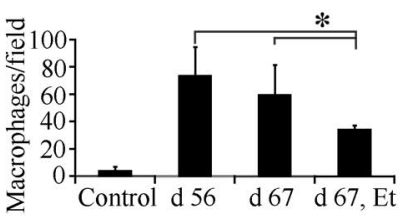

G

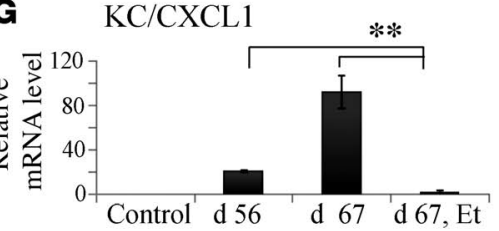

H $\quad$ MCP-1/CCL2

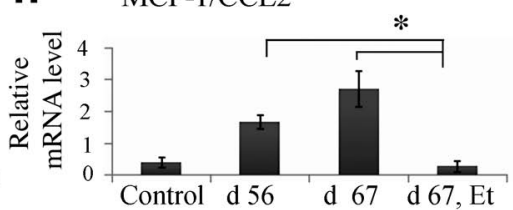

Figure 6

The effects of a TNF antagonist, etanercept, on colon carcinogenesis. (A) Schematic overview of etanercept administration. Colons were removed at day 67 after the mice were administered etanercept (Et) or a vehicle control between days 56 and 60 . (B) The tumor sizes and numbers were determined macroscopically. The bars represent the median of each group. Each symbol represents the tumor numbers of each animal or the average size of the tumors of each animal. (C) Macroscopic evaluation of the tumors. Colons were removed on day 67 from WT mice, treated with etanercept or with vehicle. Representative results from 10 independent animals are shown here. Original magnification, $\times 6$. (D) Colons were processed for hematoxylin and eosin staining and representative results from 5 independent animals are shown here. Original magnification, $\times 40$. (E and F) Myeloperoxidase- $(E)$ and F4/80-positive cells $(F)$ were enumerated as described in Methods. All values represent the mean $\pm \mathrm{SD}\left(n=10\right.$ animals). ${ }^{*} P<0.05,{ }^{* *} P<0.01$ versus etanercept-untreated WT mice. (G and $\left.\mathbf{H}\right)$ Quantitative RT-PCR analysis for KC/CXCL1 $(\mathbf{G})$ and MCP-1/CCL2 $(\mathbf{H})$ was performed on total RNAs extracted from the colons at the indicated time intervals as described in Methods. KC/CXCL1 and MCP-1/CCL2 mRNA levels were normalized to the levels of GAPDH mRNA. ${ }^{*} P<0.05$, ${ }^{* *} P<0.01$ versus etanercept untreated WT mice.

gene, particularly at its glycogen synthase kinase- $3 \beta$ (GSK-3 $\beta$ ) phosphorylation sites, mutated more frequently than adenomatous polyposis coli (APC) gene (28). Hence, we examined the effects of TNF blockade on the mutations of the GSK-3 $\beta$ phosphorylation sites of the $\beta$-catenin gene, located in its exon 3 . Mutations of $\beta$-catenin gene were detected in all tumors, and etanercept treatment reduced the mutation frequency markedly from $10 / 10$ positive in the untreated group to $3 / 10$ in the etanercept-treated group (Supplemental Table 1). These observations suggest that blocking of TNF signaling can reverse tumorigenesis even when colon carcinoma is already present, probably by reducing the infiltration of inflammatory cells. Such cells are a major source of COX-2, which is presumed to be involved in both tumor neovascularization and $\beta$-catenin activation.

\section{Discussion}

During relapses acute attacks of UC cause a massive infiltration of neutrophils and mononuclear cells into the lamina propria and ulceration of colon extending into the submucosa. During remissions of active disease, granulation tissues fill the ulcer craters, accompanied by regeneration of the mucosal epithelium $(1,29)$.
Aminosalicylates, corticosteroids, and cyclosporine are all used to induce remissions and prevent relapse of quiescent disease (29). Despite these medical treatments, UC patients frequently experience relapses and sometimes undergo colectomy. Moreover, recurrent relapses can cause dysplasia of regenerated mucosal epithelium and subsequent progression to frank carcinoma $(2,3)$. Accordingly, new treatments for UC are needed. A recent clinical trial demonstrated that humanized anti-TNF- $\alpha$ antibody was beneficial for the treatment of moderately to severely active UC patients (30). However, the pathogenic role of TNF- $\alpha$ in UC remains unclear and is particularly so in UC-associated colon carcinogenesis. Hence, we examined the process of chronic colitis-induced colon carcinogenesis by using mice deficient in TNF-Rp55, a major receptor for TNF- $\alpha$ as well as a TNF antagonist, and demonstrated the critical role of the TNF signaling pathway in colon carcinogenesis.

Greten and colleagues have revealed the crucial involvement of the IкB kinase $\beta / N F-\kappa B$ (IKK $\beta / N F-\kappa B$ ) in colon carcinogenesis induced by combined treatment with AOM and DSS (9). They further demonstrated that depletion of IKK $\beta$ in intestinal epithelial cells increased epithelial apoptosis and concomitantly decreased tumor incidence without affecting tumor size and inflammation. 



\section{Figure 7}

The effect of etanercept on COX-2 expression and angiogenesis. (A) Quantitative RT-PCR analysis for COX-2 was performed on total RNAs extracted from the colons at the indicated time intervals as described in Methods. The levels of COX-2 mRNA were normalized to the levels of GAPDH mRNA. ${ }^{*} P<0.05$ versus untreated (control) mice. (B and $\mathbf{C}$ ) Immunohistochemical analysis with anti-COX-2 antibody was performed on colons from WT mice as described in Methods. Boxed area in B is shown at higher magnification. Representative results from 5 independent animals are shown in $\mathbf{B}$ (original magnification, $\times 400 ; \times 1,000$ [insets]). The numbers of COX-2 expressing cells were determined on 5 independent animals as described in Methods. The mean and SD were calculated on all values and are shown in $\mathbf{C} .{ }^{*} P<0.05$ versus untreated mice. (D and $\mathbf{E}$ ) Colon tissues were immunostained with anti-CD31 antibody as described in Methods. Representative results from 5 independent animals are shown in D. Arrows in D indicate capillary vessels. Original magnification, $\times 400$. The vascular densities were determined as described in Methods and are shown in E. All values represent the mean \pm SD. ${ }^{*} P<0.05$ versus untreated mice.

In contrast, depleting IKK $\beta$ in myeloid cells reduced tumor size and inflammation without affecting apoptosis (9). However, in our model, epithelial cell apoptosis was transiently increased at day 7 in WT mice, and this increase was absent in TNF-Rp55/- mice (Supplemental Figure 2). Moreover, in the absence of TNF-Rp55, colonic inflammation was reduced as was the tumor incidence and size. Furthermore, both TNF- $\alpha$ and TNF-Rp55 were expressed mainly by infiltrating cells but not epithelial cells. Thus, it is plausible to speculate that endogenously produced TNF- $\alpha$ activated


autocrine/paracrine manner, and caused extensive colonic inflammation, thereby inducing carcinogenesis in this model.

In most cases of colon carcinogenesis, the earliest event is the loss of the APC gene, resulting in nuclear $\beta$-catenin accumulation (31), as we observed in tumor tissues in this study. Carcinoma progres- chemokines ization. Furthermore, some of these Because TNF- $\alpha$ can induce the expression of these chemokines in vitro and in vivo $(23,24)$, the TNF- $\alpha$ /TNF receptor axis may regulate the angiogenic switch directly or indirectly by inducing the expression of chemokines, which can induce both the proliferation of endothelial cells and the infiltration of inflammatory cells, another rich source of angiogenic factors.

Epidemiological studies demonstrated that nonsteroidal antiinflammatory drugs (NSAIDs) are effective in reducing the incidence of colon cancer $(41,42)$. NSAIDs can inhibit the enzymatic activities of both COX-1 and COX-2, but only COX-2 expression was enhanced in colon carcinoma tissues (43). Moreover, COX-2 gene ablation or the administration of a selective COX-2 inhibitor suppressed the intestinal polyposis observed in APC-deficient mice (44). COX-2 converts arachidonic acid to $\mathrm{PGH}_{2}$, which is 


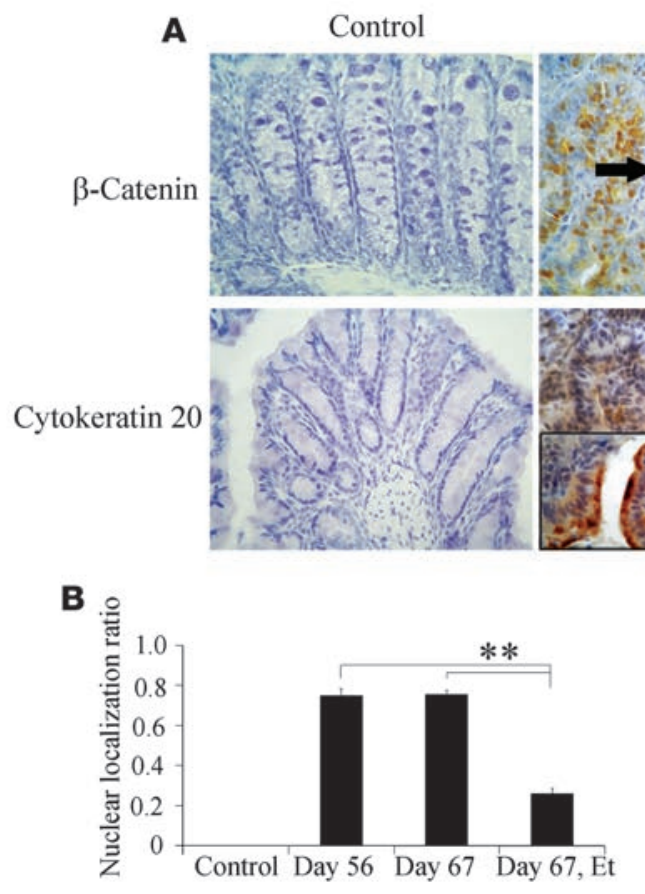

Day 56

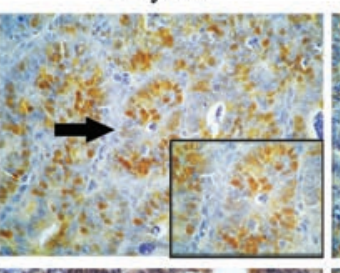

Day 67



Day 67 , Et

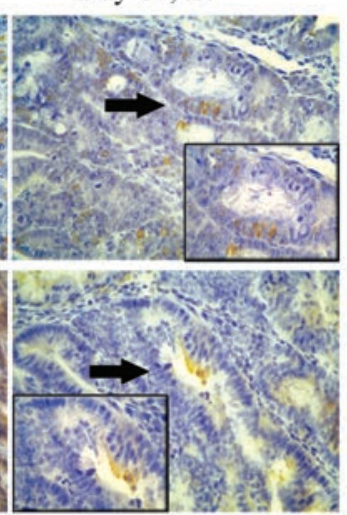

c

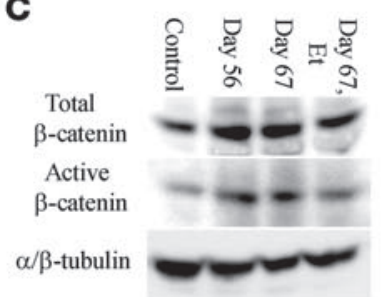

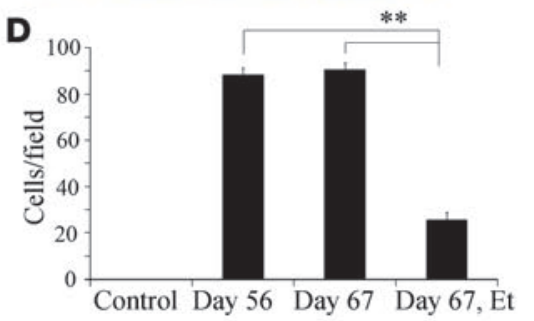

\section{Figure 8}

The effect of etanercept on $\beta$-catenin nuclear translocation. (A) Colons were immunostained with anti- $\beta$-catenin (upper panels) or anti-cytokeratin 20 antibody (lower panels) and representative results from 5 independent animals are shown. Insets are higher magnifications of the positively stained cells, indicated by arrows. Original magnification, $\times 400 ; \times 1,000$ (insets). (B) The $\beta$-catenin nuclear localization ratio was determined as the ratio of the numbers of tumor nuclei with $\beta$-catenin localization to the total number of tumor nuclei per field. At least 5 randomly chosen fields at $\times 400$ magnification were examined. All values represent the mean \pm SD. ${ }^{* *} P<0.01$ versus etanercept untreated WT mice. (C) Immunoblotting analysis with anti- $\beta$-catenin antibodies was performed on cell lysates from colon tissues as described in Methods. Representative results from 3 independent experiments are shown here. (D) The numbers of cytokeratin 20-positive cells were determined on 5 randomly chosen visual fields at $\times 400$ magnification. All values represent the mean \pm SD. ${ }^{\star \star} P<0.01$ versus etanercept untreated WT mice.

further converted to other PGs including $\mathrm{PGE}_{2}$ and thromboxanes (25). Several lines of evidence implicate $\mathrm{PGE}_{2}$ as an essential mediator for angiogenesis in colon carcinogenesis (25, 45-47). In our model, we also observed that COX- 2 mRNA expression was enhanced by DSS ingestion and that COX-2 proteins were detected in F4/80-positive macrophages and Ly-6G-positive neutrophils, similar to what has been observed in human and mouse colon cancer tissues $(43,48,49)$. Moreover, TNF-Rp55 gene ablation and TNF antagonist administration reduced both COX-2 expression and tumor angiogenesis. Thus, TNF may also enhance tumor angiogenesis by inducing the infiltration of COX-2expressing macrophages and neutrophils.

Accumulating evidence suggests that $\mathrm{PGE}_{2}$ has direct effects in enhancing colonic epithelial cell survival. $\mathrm{PGE}_{2}$ transactivated nuclear peroxisome proliferator-activated receptor $\delta$ through $\mathrm{PI} 3 \mathrm{~K} /$ Akt signaling, resulting in promoted cell survival and intestinal adenoma formation (50). Moreover, $\mathrm{PGE}_{2}$ transactivated hepatocyte growth factor (HGF) receptor and induced urokinase-type plasminogen activator receptor $\mathrm{mRNA}$, thereby enhancing colon cancer cell invasive capacity (51). It is of interest that $\mathrm{PGE}_{2}$-induced HGF receptor transactivation also induced nuclear translocation of $\beta$-catenin (51), suggesting a connection between $\mathrm{PGE}_{2}$ and the Wnt $/ \beta$-catenin axis. Supporting this notion, upon binding to its specific receptor, EP2, $\mathrm{PGE}_{2}$ activated the $\mathrm{Wnt} / \beta$-catenin axis in colon cancer cells through the phosphoinositide 3-kinase/Akt-G protein $\alpha_{s}$-axin signaling axis resulting in the promotion of cell growth (26). Moreover, $\mathrm{PGE}_{2}$ increased the phosphorylation of GSK- 3 and induced the accumulation of $\beta$-catenin and the expression of its transcription partner, $\mathrm{T}$ cell factor-4, thereby inducing the $\beta$-catenin/T cell factor-dependent gene transcription in colon cancer cells (52). Thus, COX-2-derived $\mathrm{PGE}_{2}$ can directly regulate colon carcinogenesis by regulating Wnt signaling pathway, which has a crucial role in colon carcinogenesis. We observed, on the other hand, that etanercept consistently reduced COX-2 expression and the amount of nuclear $\beta$-catenin as well as neovascularization, even when it was administered after macroscopic tumor formation. Therefore, TNF blocking may retard the progression of colon carcinomas by reducing COX-2 expression and eventually inhibiting the Wnt signaling pathway.

Our present observations have revealed the crucial involvement of TNF- $\alpha$ in the initiation of chronic inflammation-mediated colon carcinogenesis. Moreover, blocking of TNF- $\alpha$ reversed carcinoma progression, even after colon carcinoma was established. TNF- $\alpha$ blocking agents can have serious adverse effects including the induction of bacterial, tuberculosis, and opportunistic infections, but the incidence is low $(30,53)$. Thus, drugs targeting TNF- $\alpha$ may be useful for the treatment of cancers, particularly those arising from chronic inflammation.

\section{Methods}

Reagents and antibodies. AOM and DSS (MW 36,000-50,000) were purchased from Sigma-Aldrich and MP Biochemicals Inc., respectively. A specific 
TNF antagonist, etanercept, was purchased from Wyeth Pharmaceutical Japan. Rat anti-F4/80, rat anti-CD3, and rat anti-DEC205 antibodies were obtained from Serotec. Goat anti-COX-2 antibodies and rabbit anti-myeloperoxidase antibodies were obtained from Santa Cruz Biotechnology and Neomarkers, respectively. Rabbit anti- $\beta$-catenin, anti-active $\beta$-catenin, and rabbit anti- $\alpha / \beta$-tubulin antibodies were from Sigma-Aldrich, Upstate, and Cell Signaling Technology Inc., respectively. Rabbit polyclonal anti-TNFRp55, mouse monoclonal anti-human TNF- $\alpha$, rabbit polyclonal antiCD31, and mouse monoclonal antibody to cytokeratin 20 were obtained from Abcam. Rabbit polyclonal anti-TNF- $\alpha$ antibody was prepared by immunizing rabbits with a recombinant mouse TNF- $\alpha$ protein (54). ImmunoPure peroxidase-conjugated goat anti-mouse and goat anti-rabbit antibodies were obtained from Pierce Biotechnology Inc. Alexa Fluor 546 donkey anti-goat and Alexa Fluor 488 donkey anti-rat antibodies were obtained from Molecular Probes. Other antibodies were obtained from BD Bioscience - Pharmingen unless otherwise indicated.

Animal experiments. Pathogen-free 8- to 12-week old female WT BALB/c mice and TNF-Rp55 $5^{-/}$mice on a BALB/c genetic background, were housed under specific pathogen-free conditions with free access to food and water during the course of experiments $(16,19,20)$. Mice were injected intraperitoneally with $12 \mathrm{mg} / \mathrm{kg}$ body weight of AOM dissolved in physiological saline. Five days later, $2 \%$ DSS was given in the drinking water over 5 days, followed by 16 days of regular water. This cycle was repeated a total of 3 times (Figure 1A). Body weight was measured every week, and the animals were sacrificed at the indicated time intervals for macroscopical inspection, histological analysis, and total RNA extraction. In some experiments, WT mice were injected with etanercept at a dose of $3 \mathrm{mg} / \mathrm{kg}$ body weight every day from day 56 to day 60 (Figure 6A). All animal experiments were approved by the Committee on Animal Experimentation of Kanazawa University and performed in compliance with the university's Guidelines for the Care and Use of Laboratory Animals.

Bone marrow chimeric mice generation. Cell suspensions from male WT or TNF-Rp55/- bone marrow were prepared from femurs and tibias, filtered, and counted. Female WT or TNF-Rp55/- mice received a single intravenous injection of $1 \times 10^{7}$ bone marrow cells, after being irradiated with 8.5-Gy followed by 4.25-Gy x-rays (MPR-1520R; Hitachi) 4 hours later. The following groups of chimeric mice were generated: WT to WT, WT to


Genomic DNA was extracted from blood, and bone marrow chimerism was determined 4 weeks later by PCR for the Y chromosome-linked Sry gene (forward, 5'-TGGGACTGGTGACAATTGTC-3'; reverse, 5'-GAGTACAGGTGTGCAGCTCT-3').

Histopathological and immunobistochemical analyses of mouse colon tissues. Resected mouse colon tissues were fixed in $10 \%$ formalin neutral buffer solution (Wako) for paraffin embedding or were immediately frozen in Tissue-Tek O.C.T. compound (Sakura Fine Technical Co.) and stored at $-80^{\circ} \mathrm{C}$. Paraffin-embedded sections were cut at $5 \mu \mathrm{m}$ and stained with hematoxylin and eosin solution. Paraffin-embedded sections were additionally deparaffinized for immunohistochemical detection of cells positively stained for $\beta$-catenin, TNF-Rp55, MPO, F4/80, CD3, CD31, or cytokeratin 20. Frozen sections were fixed in $4 \%$ paraformaldehyde/PBS for 15 minutes for immunohistochemical detection of cells positively stained for mouse TNF- $\alpha, \mathrm{CD} 4, \mathrm{CD} 8$, or DEC205. Endogenous peroxidase activity was blocked using $3 \% \mathrm{H}_{2} \mathrm{O}_{2}$ for 5 minutes, followed by incubation with Non-Specific Staining Blocking reagent (DakoCytomation) for $10 \mathrm{~min}-$ utes. The sections were incubated with the optimal dilutions of anti-MPO, anti-F4/80, anti-CD3, anti-CD4, anti-CD8, anti-DEC205, anti- $\beta$-catenin, anti-COX-2, anti-CD31, or anti-cytokeratin 20 antibodies overnight at $4{ }^{\circ} \mathrm{C}$. MPO-, $\beta$-catenin-, TNF- $\alpha-$, TNF-Rp55-, and anti-CD31-positive cells were detected with HRP-labeled anti-rabbit polymer (EnVision+ Sys- tem; DakoCytomation), while F4/80- and CD3-positive cells were detected using Catalyzed Signal Amplification (CSA) System (DakoCytomation). Immune complexes were visualized with Peroxidase Substrate DAB kit (Vector Laboratories Inc.). CD4-, CD8-, DEC205-, and COX-2-positive cells were detected by the incubation with anti-rat or anti-goat biotinylated IgG (1:200; DakoCytomation). Detection of cytokeratin 20 was performed with Vector M.O.M. Immunodetection kit (Vector Laboratories Inc.), which was used according to the manufacturer's instructions. The resultant immune complexes were visualized by $\mathrm{ABC}$ Elite kit (Vector Laboratories Inc.) and Peroxidase Substrate DAB kit (Vector Laboratories Inc.) according to the manufacturer's instructions. Finally, the slides were counterstained with hematoxylin, dehydrated, and coverslipped. Positive cells were enumerated on 5 randomly chosen visual fields at $\times 400$ magnification. The pixel numbers of CD31-positive areas were measured on 5 randomly chosen visual fields at $\times 200$ magnification with the aid of Adobe Photoshop software. For double-color immunofluorescence analysis, the sections were incubated with the combination of anti-COX-2 and anti-F4/80 or with anti-COX-2 and anti-Ly-6G antibodies at $4{ }^{\circ} \mathrm{C}$, overnight. Alexa Fluor 546 donkey antigoat and Alexa Fluor 488 donkey anti-rat antibodies were used as secondary antibodies. Immunofluorescence was visualized on a Carl Zeiss Laser Scanning Microscope 510.

Immunobistochemical detection of TNF- $\alpha$ in buman colon tissues. The tissues were obtained upon biopsy from patients with UC and colorectal cancer with an informed consent and with approval from the Human Subjects Research Ethical Committee of Kanazawa University Hospital. The tissues were fixed and paraffin-embedded and were cut at $5 \mu \mathrm{m}$. Paraffin-embedded sections were additionally deparaffinized for immunohistochemical analysis, using the combination of anti-human TNF- $\alpha$ mouse monoclonal antibody and CSA system.

TUNEL assay. Paraffin-embedded sections were stained with In situ Apoptosis Detection Kit (TaKaRa Bio Inc.), according to the manufacturer's instructions, to detect apoptotic cells. TUNEL-positive cells were counted on 5 randomly chosen visual fields at $\times 400$ magnification.

Quantitative RT-PCR. Total RNA was extracted from colon tissues with RNA-Bee (Tel-Test Inc.) and $2.5 \mu \mathrm{g}$ of RNA was reverse-transcribed using ReverTraAce (Toyobo) and random primers as described previously. Realtime PCR was performed on Applied Biosystems StepOne Real-Time PCR System (Applied Biosystems) using the comparative $\mathrm{C}_{\mathrm{t}}$ quantitation method. TaqMan Gene Expression Assays (Applied Biosystems) containing specific primers (accession numbers: TNF- $\alpha-M m 00443258 \_\mathrm{m} 1, \mathrm{KC} /$ CXCL1-Mm00433859_m1, MCP-1/CCL2-Mm00441242_m1, COX-2Mm00478374_m1, GAPDH-Mm99999915_g1), TaqMan MGB probe (FAM dye-labeled), and TaqMan Fast Universal PCR Master Mix were used with $10 \mathrm{ng}$ of cDNA to detect and quantify the expression levels of TNF- $\alpha$, $\mathrm{KC} / \mathrm{CXCL} 1, \mathrm{MCP}-1 / \mathrm{CCL} 2$, and COX-2 in mouse colon tissues. GAPDH was amplified as internal control. $\mathrm{C}_{\mathrm{t}}$ values of GAPDH were subtracted from $C_{t}$ values of the target genes $\left(\Delta \mathrm{C}_{\mathrm{t}}\right)$. $\Delta \mathrm{C}_{\mathrm{t}}$ values of treated mice were compared with $\Delta \mathrm{C}_{\mathrm{t}}$ values of untreated animals. Reactions were done at $95^{\circ} \mathrm{C}$ for 20 seconds followed by 40 cycles of $95^{\circ} \mathrm{C}$ for 1 second and $60^{\circ} \mathrm{C}$ for 20 seconds, $60^{\circ} \mathrm{C}, 20$ seconds -40 cycles.

DNA sequencing and mutation analysis of $\beta$-catenin gene. After sequential treatment with AOM and DSS, WT mice were injected with either vehicle or etanercept every day from day 56 to day 60 (Figure 6A). Tumors were obtained at day 67 and embedded in paraffin. Genomic DNA was extracted from paraffin-embedded tumor sections by using NucleoSpin Tissue Kit (Macherey-Nagel Inc.) according to the manufacturer's instructions. Exon 3 of the $\beta$-catenin gene, containing the consensus sequence for GSK-3 $\beta$ phosphorylation, was amplified by PCR, using specific primers (forward, 5'-GCTGACCTGATGGAGTTGGA-3', reverse, 5'-GCTACTTGCTCTTGCGTGAA- $3^{\prime}$ ) and the following thermal cycling parameters: $94^{\circ} \mathrm{C}, 5$ min- 
utes; followed by 35 cycles at $94^{\circ} \mathrm{C}, 45$ seconds, $55^{\circ} \mathrm{C}, 1$ minute, and $72^{\circ} \mathrm{C}$, 1 minute; followed by $72^{\circ} \mathrm{C}, 5$ minutes. PCR products were subcloned into pSTBlue-1 vector (AccepTor vector; Novagen) and sequenced using BigDye Terminator Ver. 3.1 Cycle Sequencing kit (Applied Biosystems) on an ABI PRISM 3100-Avant Genetic Analyzer (Applied Biosystems).

Immunoblotting analysis. Colon tissues were homogenized and sonicated in RIPA lysis buffer (Santa Cruz Biotechnology Inc.), supplemented with protease inhibitors. After centrifugation at $20,000 \mathrm{~g}$ for 15 minutes, $30 \mu \mathrm{g}$ of the supernatants were separated on $10 \%$ SDS-polyacrylamide gel and transferred onto an Immobilon-P Transfer membrane (Millipore). After being blocked with $5 \%$ skim milk, the membrane was incubated with antibodies to total $\beta$-catenin $(1: 1,000)$ and active $\beta$-catenin (1:500). Rabbit anti- $\alpha /$ $\beta$-tubulin antibody $(1: 1,000)$ was used as an internal control. ImmunoPure peroxidase-conjugated anti-mouse or anti-rabbit IgG were used as secondary antibodies. The blotted membrane was then treated with the Super Signal West Dura Extended Duration Substrate (Pierce Biotechnology Inc.) and signals were detected by LAS-3000 mini CCD camera (Fuji Film).

Statistics. The means \pm SD were calculated for all parameters determined. Statistical significance was evaluated using 1-way ANOVA, followed by Fisher's protected least significant difference test. $P$ values less than 0.05 were considered statistically significant.

1. Fiocchi, C. 1998. Inflammatory bowel disease: Etiology and pathogenesis. Gastroenterology. 115:182-205.

2. Risques, R.A., Rabinovitch, P.S., and Brentnall, T.A. 2006. Cancer surveillance in inflammatory bowel disease: new molecular approaches. Curr. Opin. Gastroenterol. 22:382-390.

3. Ullman, T., Croog, V., Harpaz, N., Sachar, D., and Itzkowitz, S. 2003. Progression of flat low-grade dysplasia to advanced neoplasia in patients with ulcerative colitis. Gastroenterology. 125:1311-1319.

4. Dobbins, W.O., 3rd. 1984. Dysplasia and malignancy in inflammatory bowel disease. Annu. Rev. Med. 35:33-48.

5. Okayasu, I., et al. 1990. A novel method in the induction of reliable experimental acute and chronic ulcerative colitis in mice. Gastroenterology. 98:694-702.

6. Okayasu, I., et al. 2002. Dysplasia and carcinoma development in a repeated dextran sulfate sodiuminduced colitis model. J. Gastroenterol. Hepatol. 17:1078-1083.

7. Boivin, G.P., et al. 2003. Pathology of mouse models of intestinal cancer: consensus report and recommendations. Gastroenterology. 124:762-777.

8. Okayasu, I., Ohkusa, T., Kajiura, K., Kanno, J., and Sakamoto, S. 1996. Promotion of colorectal neoplasia in experimental murine ulcerative colitis. Gut. 39:87-92.

9. Greten, F.R., et al. 2004. IKK $\beta$ links inflammation and tumorigenesis in a mouse model of colitisassociated cancer. Cell. 118:285-296.

10. Lind, D.S., et al. 2001. NF- $\mathrm{KB}$ is upregulated in colorectal cancer. Surgery. 130:363-369.

11. Hardwick, J.C., van den Brink, G.R., Offerhaus, G.J., van Deventer, S.J., and Peppelenbosch, M.P. 2001. NF-KB, p38 MAPK and JNK are highly expressed and active in the stroma of human colonic adenomatous polyps. Oncogene. 20:819-827.

12. Li, Q., Withoff, S., and Verma, I.M. 2005. Inflammation-associated cancer: NF-kappaB is the lynchpin. Trends Immunol. 26:318-325.

13. Liu, Z.G. 2005. Molecular mechanism of TNF signaling and beyond. Cell Res. 15:24-27.

14. Tracey, K.J. 1994. Tumor necrosis factor-alpha. In The cytokine handbook. 2nd edition. A. Thomson, editor. Academic Press. London, United Kingdom. 289-304.

15. Pfeffer, K., et al. 1993. Mice deficient for the 55 $\mathrm{kd}$ tumor necrosis factor receptor are resistant to endotoxic shock, yet succumb to L. monocytogenes

\section{Acknowledgments}

We would like to express our gratitude to Joost J. Oppenheim (National Cancer Institute-Frederick) for his critical review and editing of the manuscript.

Received for publication April 20, 2007, and accepted in revised form November 28, 2007.

Address correspondence to: Naofumi Mukaida, Division of Molecular Bioregulation, Cancer Research Institute, Kanazawa University, 13-1 Takara-machi, Kanazawa 920-0934, Japan. Phone: 81-76-265-2767; Fax: 81-76-234-4520; E-mail: naofumim@ kenroku.kanazawa-u.ac.jp.

Kazuya Kitamura's present address is: Mucosal Immunology Section, Laboratory of Molecular Immunology, NIAID, NIH, Bethesda, Maryland, USA.

Chifumi Fujii's present address is: Department of Integrative Physiology and Biosystem Control, Graduate School of Medicine, Shinshu University, Matsumoto, Japan.

infection. Cell. 73:457-467.

16. Mori, R., Kondo, T., Oshima, T., Ishida, Y., and Mukaida, N. 2002. Accelerated wound healing in tumor necrosis factor receptor p55-deficient mice with reduced leukocyte infiltration. FASEB J. 16:963-974.

17. Bazzoni, F., and Beutler, B. 1996. The tumor necrosis factor ligand and receptor families. N. Engl. J. Med. 334:1717-1725.

18. Senzer, N., et al. 2004. TNFerade biologic, an adenovector with a radiation-inducible promoter, carrying the human tumor necrosis factor alpha gene: a phase I study in patients with solid tumors. J. Clin. Oncol. 22:592-601.

19. Kitakata, H., et al. 2002. Essential roles of tumor necrosis factor receptor p55 in liver metastasis of intrasplenic administration of colon 26 cells. Cancer Res. 62:6682-6687.

20. Tomita, Y., et al. 2004. Spontaneous regression of lung metastasis in the absence of tumor necrosis factor receptor p55. Int. J. Cancer. 112:927-933.

21. Moore, R.J., et al. 1999. Mice deficient in tumor necrosis factor- $\alpha$ are resistant to skin carcinogenesis. Nat. Med. 5:828-831.

22. Liu, R., Bal, H.S., Desta, T., Behl, Y., and Graves, D.T. 2006. Tumor necrosis factor- $\alpha$ mediates diabetes-enhanced apoptosis of matrix-producing cells and impairs diabetic healing. Am. J. Pathol. 168:757-764.

23. Shiratori, Y., et al. 1994. Modulation of KC/gro protein (interleukin- 8 related protein in rodents) release from hepatocytes by biologically active mediators. Biochem. Biophys. Res. Commun. 203:1398-1403.

24. Marra, F., Valente, A.J., Pinzani, M., and Abboud, H.E. 1993. Cultured human liver fat-storing cells produce monocyte chemotactic protein-1. Regulation by proinflammatory cytokines. J. Clin. Invest. 92:1674-1680.

25. Iniguez, M.A., Rodriguez, A., Volpert, O.V., Fresno, M., and Redondo, J.M. 2003. Cyclooxygenase-2: a therapeutic target in angiogenesis. Trends Mol. Med. 9:73-78.

26. Castellone, M.D., Teramoto, H., Williams, B.O., Druey, K.M., and Gutkind, J.S. 2005. Prostaglandin $\mathrm{E}_{2}$ promotes colon cancer cell growth through a $G_{s}$-axin- $\beta$-catenin signalling axis. Science. 310:1504-1510.

27. Berezowski, K., Stastny, J.F., and Kornstein, M.J. 1996. Cytokeratins 7 and 20 and carcinoembryonic antigen in ovarian and colonic carcinoma. Mod.
Pathol. 9:426-429

28. Takahashi, M., and Wakabayashi, K. 2004. Gene mutations and altered gene expression in azoxymethane-induced colon carcinogenesis in rodents. Cancer Sci. 95:475-480.

29. Hanauer, S.B. 2004. Medical therapy for ulcerative colitis 2004. Gastroenterology. 126:1582-1592.

30. Rutgeerts, P., et al. 2005. Infliximab for induction and maintenance therapy for ulcerative colitis. N. Engl. J. Med. 353:2462-2476.

31. Takayama, T., et al. 2001. Analysis of K-ras, APC, and beta-catenin in aberrant crypt foci in sporadic adenoma, cancer, and familial adenomatous polyposis. Gastroenterology. 121:599-611.

32. Babbar, N., and Casero, R.A., Jr. 2006. Tumor necrosis factor- $\alpha$ increases reactive oxygen species by inducing spermidine oxidase in human lung epithelial cells: a potential mechanism for inflammation-induced carcinogenesis. Cancer Res. 66:11125-11130.

33. Yan, B.Y., et al. 2006. Tumor necrosis factor- $\alpha$ is a potent endogenous mutagen that promotes cellular transformation. Cancer Res. 66:11565-11570.

34. Scott, K.A., et al. 2003. An anti-tumor necrosis factor- $\alpha$ antibody inhibits the development of experimental skin tumors. Mol. Cancer Ther. 2:445-451.

35. Bergers, G., and Benjamin, L.E. 2003. Tumorigenesis and the angiogenic switch. Nat. Rev. Cancer. 3:401-410.

36. Yoshida, S., et al. 1997. Involvement of interleukin-8, vascular endothelial growth factor, and basic fibroblast growth factor in tumor necrosis factor alpha-dependent angiogenesis. Mol. Cell. Biol. 17:4015-4023.

37. Leibovich, S.J., et al. 1987. Macrophage-induced angiogenesis is mediated by tumour necrosis factor- $\alpha$. Nature. 329:630-632.

38. Lin, E.Y., et al. 2006. Macrophages regulate the angiogenic switch in a mouse model of breast cancer. Cancer Res. 66:11238-11246.

39. Nozawa, H., Chiu, C., and Hanahan, D. 2006. Infiltrating neutrophils mediate the initial angiogenic switch in a mouse model of multistage carcinogenesis. Proc. Natl. Acad. Sci. U. S. A. 103:12493-12498.

40. Bernardini, G., et al. 2003. Analysis of the role of chemokines in angiogenesis. J. Immunol. Methods. 273:83-101.

41. Kune, G.A., Kune, S., and Watson, L.F. 1988. Colorectal cancer risk, chronic illnesses, operations, and medications: case control results from 
the Melbourne colorectal cancer study. Cancer Res. 48:4399-4404.

42. Thun, M.J., Nanboodiri, M.M., and Heath, C.W. 1991. Aspirin use and reduced risk of fatal colon cancer. N. Engl. J. Med. 325:1593-1596.

43. Sano, H., et al. 1995. Expression of cyclooxygenase-1 and -2 in human colorectal cancer. Cancer Res. 55:3785-3789.

44. Oshima, M., et al. 2002. Suppression of intestinal polyposis in $\mathrm{Apc}^{\Delta 716}$ knockout mice by inhibition of cyclooxygenase 2 (COX-2). Cell. 87:803-809.

45. Sonoshita, M., et al. 2001. Acceleration of intestinal polyposis through prostaglandin receptor $\mathrm{EP}_{2}$ in $\mathrm{Apc}^{\Delta 716}$ knockout mice. Nat. Med. 7:1048-1051.

46. Seno, H., et al. 2002. Cyclooxygenase-2 and prostaglandin $\mathrm{E}_{2}$ receptor $\mathrm{EP}_{2}$-dependent angiogen- esis in $\mathrm{Apc}^{\Delta 716}$ mouse intestinal polyps. Cancer Res. 62:506-511.

47. Mutoh, M., et al. 2002. Involvement of prostaglandin $\mathrm{E}$ receptor subtype $\mathrm{EP}_{4}$ in colon carcinogenesis. Cancer Res. 62:28-32.

48. Hull, M.A., Tisbury, A., Scott, N., Bonifer, C., and Coletta, P.L. 1999. Cyclooxygenase-2 is up-regulated and localized to macrophages in the intestine of Min mice. Brit. J. Cancer. 79:1399-1405.

49. Bamba, H., et al. 1999. High expression of cyclooxygenase- 2 in macrophages of human colonic adenoma. Int. J. Cancer. 83:470-475.

50. Wang, D., et al. 2004. Prostaglandin $\mathrm{E}_{2}$ promotes colorectal adenoma growth via transactivation of the nuclear peroxisome proliferators-activated receptor $\delta$. Cancer Cell. 6:285-295.
51. Pai, R., Nakamura, T., Moon, W.S., and Tarnawski, A.S. 2003. Prostaglandins promote colon cancer cell invasion; signaling by cross-talk between two distinct growth factor receptors. FASEB J. 17:1640-1647.

52. Shao, J., Jung, C., Liu, C., and Sheng, H. 2005. Prostaglandin $\mathrm{E}_{2}$ stimulates the $\beta$-catenin/T cell factor-dependent transcription in colon cancer. J. Biol. Chem. 280:26565-26572.

53. Botsios, C. 2005. Safety of tumour necrosis factor and interleukin-1 blocking agents in rheumatic diseases. Autoimmun. Rev. 4:162-170.

54. Fujioka, N., et al. 1995. Preparation of specific antibodies against murine IL-1ra and the establishment of IL-1ra as an endogenous regulator of bacteria-induced fulminant hepatitis in mice. J. Leukoc. Biol. 58:90-98. 